\title{
On the excitation of ULF waves by solar wind pressure enhancements
}

\author{
P. T. I. Eriksson ${ }^{1}$, L. G. Blomberg ${ }^{1}$, S. Schaefer ${ }^{2}$, and K.-H. Glassmeier ${ }^{2}$ \\ ${ }^{1}$ Space and plasma physics, School of Electrical Engineering, Royal Institute of Technology, Stockholm, Sweden \\ ${ }^{2}$ Institute for Geophysics and Extraterrestrial Physics, Technical University of Braunschweig, Braunschweig, Germany
}

Received: 14 June 2006 - Revised: 18 September 2006 - Accepted: 13 October 2006 - Published: 22 November 2006

\begin{abstract}
We study the onset and development of an ultra low frequency (ULF) pulsation excited by a storm sudden commencement. On 30 August 2001, 14:10 UT, the Cluster spacecraft are located in the dayside magnetosphere and observe the excitation of a ULF pulsation by a threefold enhancement in the solar wind dynamic pressure. Two different harmonics are observed by Cluster, one at $6.8 \mathrm{mHz}$ and another at $27 \mathrm{mHz}$. We observe a compressional wave and the development of a toroidal and poloidal standing wave mode. The toroidal mode is observed over a narrow range of L-shells whereas the poloidal mode is observed to have a much larger radial extent. By looking at the phase difference between the electric and magnetic fields we see that for the first two wave periods both the poloidal and toroidal mode are travelling waves and then suddenly change into standing waves. We estimate the azimuthal wave number for the $6.8 \mathrm{mHz}$ to be $m=10 \pm 3$. For the $27 \mathrm{mHz}$ wave, $m$ seems to be several times larger and we discuss the implications of this. We conclude that the enhancement in solar wind pressure excites eigenmodes of the geomagnetic cavity/waveguide that propagate tailward and that these eigenmodes in turn couple to toroidal and poloidal mode waves. Thus our observations give firm support to the magnetospheric waveguide theory.
\end{abstract}

Keywords. Magnetospheric physics (MHD waves and instabilities; Solar wind-magnetosphere interactions)

\section{Introduction}

Storm sudden commencements (SSC) and sudden impulses (SI) are generated by steep increases in the solar wind dynamic pressure. They are often followed by ULF wave activity. The magnetosphere's response to a sudden increase in

Correspondence to: P. T. I. Eriksson

(tommy.eriksson@ee.kth.se) the solar wind dynamic pressure is often described by what is observed by ground based magnetometers (e.g. Chi et al., 2001, and references therein). Because of the solar wind dependence, the number of yearly SSC events is correlated with the solar cycle. The study of ULF waves in conjunction with SSCs or SIs is tractable from at least two vantage points. One is that unlike other excitation mechanisms the specific source of energy for the waves is clear. Second, the response of any system to an impulse or step-like function yields the eigenmodes of the system. Thus these events serve as a diagnostic tool for the magnetosphere.

Radoski (1974) and also Kivelson and co-workers (Kivelson et al., 1984; Kivelson and Southwood, 1985, 1986) showed that the magnetosphere can act as a cavity in response to disturbances at the magnetopause. Walker et al. (1992) noted that since the magnetosphere is open downtail, a waveguide would be a better description. This waveguide description has been extensively investigated in a box-model geometry by Wright (1994) and by Wright and Rickard (1995a, b). Mann et al. (1999) studied how the reflection of waves inside the magnetosphere is affected by different magnetosheath flow speeds. They showed that for high solar wind speeds the magnetopause can effectively trap and energize waveguide-modes in the magnetosphere. In a complementary study to that of Mann et al. (1999), Walker (1998) investigated waves incident from outside the magnetosphere. Neither of these studies included the effects of coupling to a resonant field line (Tamao, 1966; Chen and Hasegawa, 1974a; Southwood, 1974). In a recent paper, Walker (2005) included the resonance region and studied how an external source, such as a quasi-monochromatic wave or a broadband disturbance, can excite magnetospheric oscillations and thus also field line resonances (FLRs). They show that if the source at the magnetopause contains energy at a frequency coinciding with a normal mode of the waveguide that mode will be excited. This result applies to both monochromatic waves and broadband perturbations.

Published by Copernicus GmbH on behalf of the European Geosciences Union. 


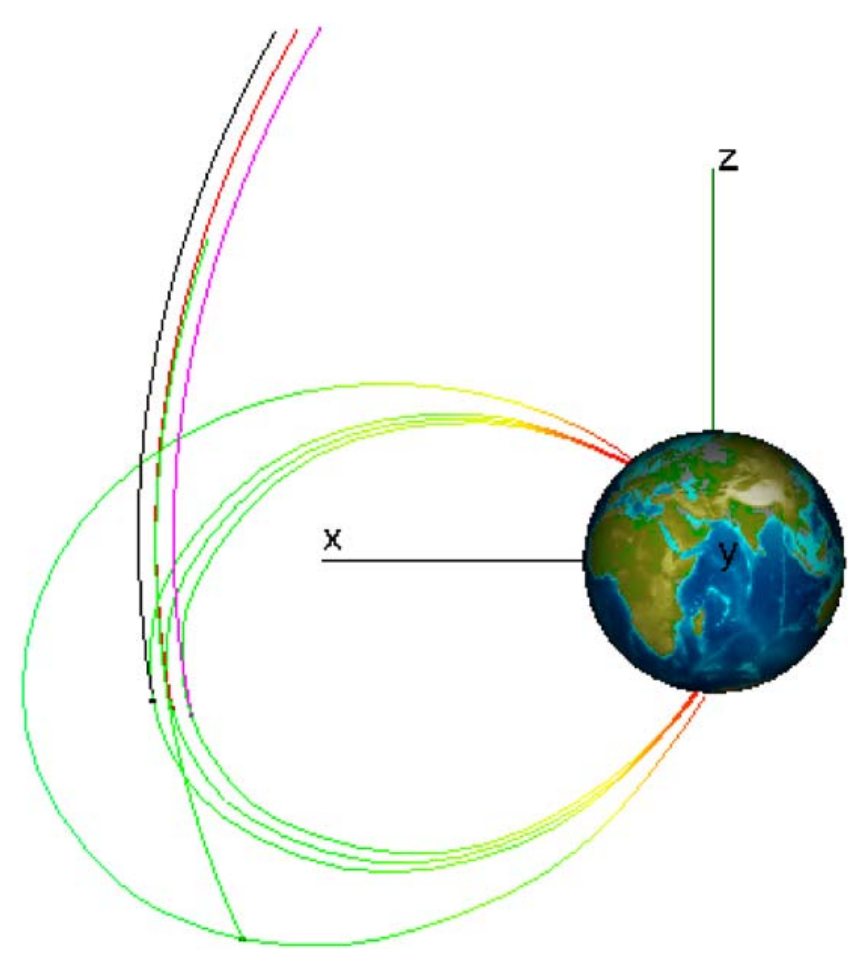

Fig. 1. Illustration of the spacecraft orbit in GSM coordinates. The spacecraft positions are shown at 14:10 UT and the field lines are traced using the T89 model. The orbits are drawn from 14:10 UT to $16: 10$ UT. Positive $x$ is to the left; positive $z$ up; and positive $y$ is out of the papers plane.

In addition to modelling steady state excitation of the magnetospheric cavity/waveguide, several authors have studied impulsive excitation. In the case of a cavity model, Allan et al. (1986) studied the response of a cylindrical model of the magnetosphere to a compressional impulse at the magnetopause. Chen and Hasegawa (1974b) and Southwood and Kivelson (1990) used a box-model of the magnetosphere. Lee and Lysak $(1989,1991)$ used a dipole model for the magnetic field. Although using different models, these authors present the same basic picture of the response of the magnetosphere to a pressure impulse impinging on the magnetopause: The impulse excites several monochromatic compressional cavity eigenmodes. At locations where the local field-line eigenperiod matches the cavity eigenperiods these compressional waves leak energy to field line resonances. Goldstein et al. (1999) used a model similar to that of Lee and Lysak (1989) to simulate Polar satellite observations of ULF waves related to a solar wind density pulse.

Experimental observations of magnetospheric cavity/waveguide mode oscillations are sparse. Waters et al. (2002) treat some of the difficulties involved in detecting these modes using spacecraft data. However there are some exceptions. Mann et al. (1998) report a multisatellite observation of a compressional waveguide mode. They found this global mode to have a small azimuthal wave number $(|m| \leq 5)$ and likely to have been excited by an enhancement in the solar wind pressure. The wave was found to propagate anti-sunward and was only observed for approximately five wave cycles. There is also compelling evidence of magnetopause oscillations caused by a Kelvin-Helmholtz instability creating magnetospheric waveguide modes that excite discrete field line resonances (Mann et al., 2002; Rae et al., 2005).

A number of satellite observations of SSC excited ULF waves exist (Baumjohann et al., 1984; Cahill et al., 1990; Nopper et al., 1982). Cahill et al. (1990) observed a damped toroidal mode standing wave at $L=4.5$. At the same time they observed a compressional wave with the same period $(100 \mathrm{~s})$ as the toroidal wave and interpreted this as a global cavity mode compressional oscillation driving the toroidal mode. Shimazu et al. (1995) used both ground-based magnetometers and satellites to study a global ULF waves. They concluded that the most likely wave source was a magnetospheric cavity resonance caused by an enhancement in the solar wind dynamic pressure.

In the light of the theoretical developments of the waveguide description we present in this paper Cluster observations of ULF waves excited by an enhancement in the solar wind pressure occurring 30 August 2001. Albeit theoretically sound the waveguide description lacks firm experimental support, especially in conjunction with FLRs. This study aims at observationally verifying some of the predictions of the waveguide mode theory, but also, point out some aspects that are not fully developed yet. Combining Cluster electric and magnetic field data with the analytic signal concept (Glassmeier, 1980; Walker et al., 1992) we are able to study the azimuthal, field-aligned and radial properties of the waves as well as determining the phase difference between the electric and magnetic field components. Looking at the phase difference is important because it allows us to conclude if energy is being transported or not and in what direction.

\section{Instrumentation}

The orbits of the Cluster spacecraft are displayed in Fig. 1, for the interval 14:10 UT to 16:10 UT 30 August 2001. The Earth is plotted as reference and the field lines where the satellites are located at 14:10 UT are plotted using the Tsyganenko 89 model (Tsyganenko, 1989) with $K_{p}=1$ as input. Figure 2 gives the $L$-value (calculated using just the IGRF model), angle from the magnetic equator and magnetic local time (MLT) of the four satellites. Notice that spacecraft (SC) 1,2 and 4 are in relatively close proximity whereas SC 3 is trailing. The orbits are such that SC 1 has the largest minimum $L$-value, followed by SC 2, 3 and 4 having the smallest minimum in $L$. The satellites are also close to noon MLT. This particular configuration allows us to study the radial extent of the pulsations and also their temporal evolution. 


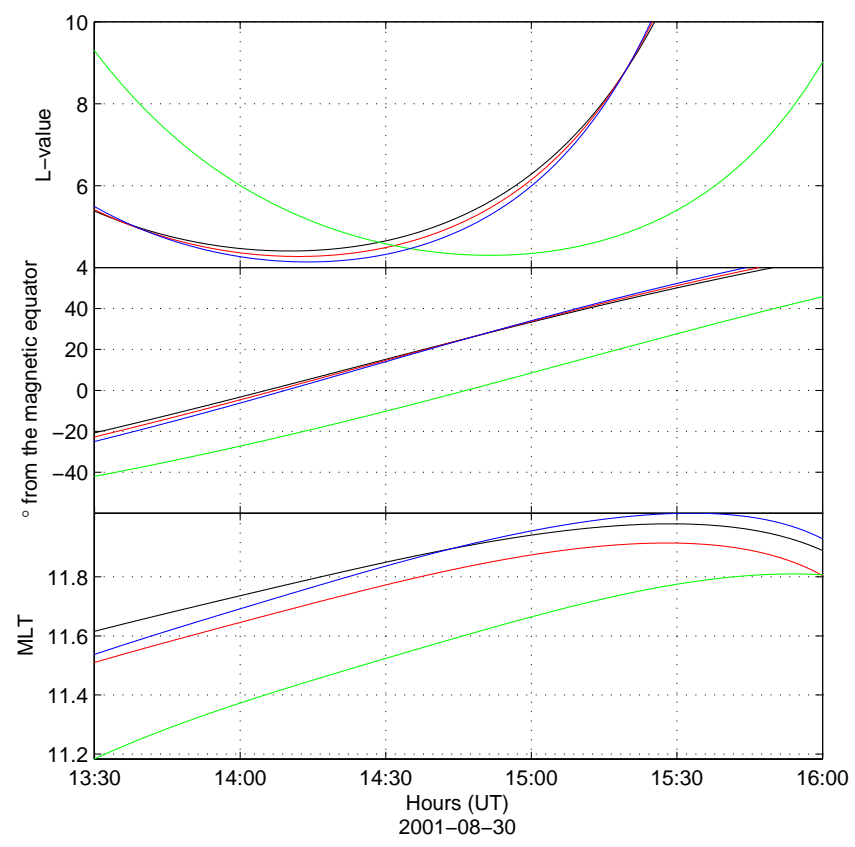

Fig. 2. $L$-value, polar angle in SM-coordinates and MLT for SC 1 (black), SC 2 (red), SC 3 (green) and SC 4 (blue).

This event occurs after a quiet period, with a minimum in Dst of $-24 \mathrm{nT}$ and $\mathrm{Kp} 1-2$ for more than $24 \mathrm{~h}$ prior to the SSC. At 14:00 UT Geotail is located upstream of the bow shock, 19.4 $R_{E}$ from Earth. From 14:08 UT to 14:10 UT Geotail observes approximately a factor of three increase in the solar wind dynamic pressure (Fig. 3). The interplanetary magnetic field rises from $5 \mathrm{nT}$ to approximately $8 \mathrm{nT}$. A storm sudden commencement follows at 14:11 UT. From the model of the equatorial electron density by Carpenter and Anderson (1992) we have calculated the inner $\left(L_{\mathrm{ppi}}\right)$ and outer $\left(L_{\mathrm{ppo}}\right)$ boundary of the plasmapause. The values are $L_{\mathrm{ppi}}=4.68$ and $L_{\mathrm{ppo}}=4.90$, respectively. Looking at Fig. 2 this would indicate that perigee of all four spacecraft is located within the plasmasphere.

\section{Observations}

Figure 4 shows the duskward electric field measured by all four spacecraft. The onset of ULF pulsations is seen on all four satellites at approximately 14:10 UT, indicated by the dashed line. There are no noticeable fluctuations in the electric field prior to the onset of the pulsations, most likely due to the long quiet period in the magnetosphere before the SSC. In this study we take no particular interest in the relative timing between observations in the solar wind and the magnetosphere but merely note that it depends on the alignment and shape of the pressure pulse impinging on the magnetosphere. Apparent is also the lack of transient wave components, in-

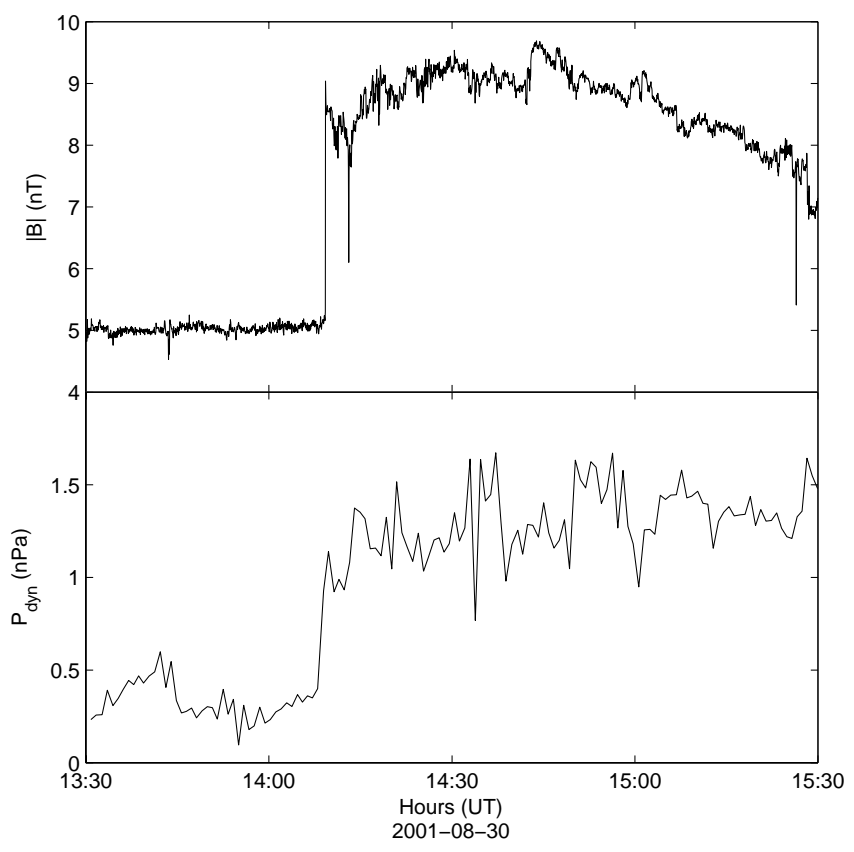

Fig. 3. Solar wind parameters measured by Geotail. The upper panel shows the absolute value of the interplanetary magnetic field. The lower panel shows the solar wind dynamic pressure.

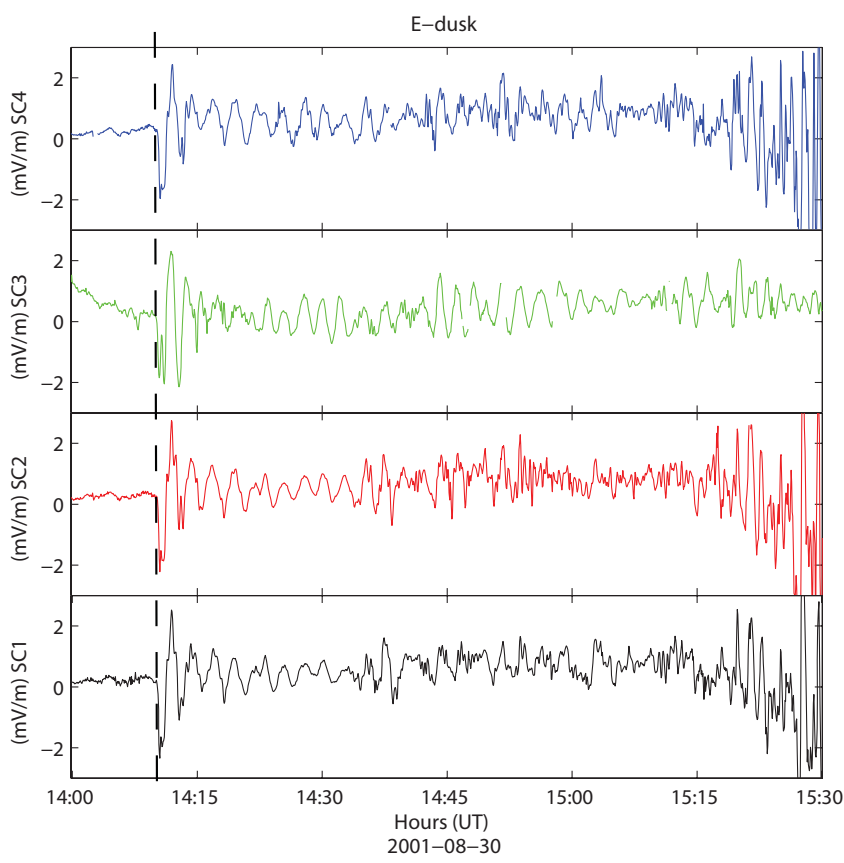

Fig. 4. Duskward electric field for for SC 1 (black), SC 2 (red), SC 3 (green) and SC 4 (blue).

stead the pulsations immediately assume a monochromatic nature.

Two discrete oscillations are observed, one at $6.8 \mathrm{mHz}$ and another at $27 \mathrm{mHz}$. By bandpass filtering the electric and 


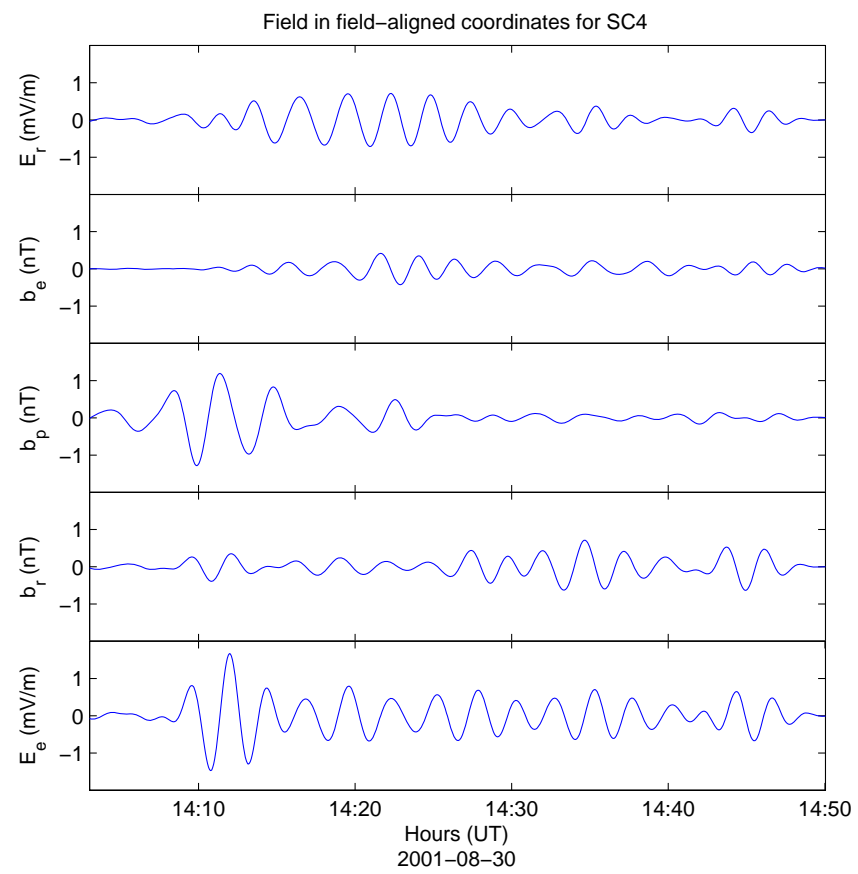

Fig. 5. Field in field-aligned coordinates measured by SC 4 . The data have been bandpass filtered in the range $4-9 \mathrm{mHz}$.

magnetic field data, using a fifth order butterworth filter, we can study the behavior of the two different harmonic frequencies separately. Figure 5 shows bandpass filtered data from $\mathrm{SC} 4$ in field-aligned coordinates. Here we use the coordinate system described by Eriksson et al. (2005b). This coordinate system has a field-aligned component $p$, a radial component $r$ and an eastward component $e$. The third component of the electric field (i.e., the spin-aligned component in spacecraft coordinates) is computed by assuming $\mathbf{E} \cdot \mathbf{B}=0$ (i.e., ideal MHD). The passband has been chosen to be 4$9 \mathrm{mHz}$ to isolate the $6.8 \mathrm{mHz}$ wave. First thing to notice is the large oscillations in the field-aligned component of the magnetic field $\left(b_{p}\right)$. Looking at the $b_{e}$ component which corresponds to the toroidal mode ( $E_{r}$ for the electric field) we can see that the amplitude of the oscillation is almost zero at the onset of the pulsation and then increases to a maximum at approximately 14:22 UT. For the $E_{r}$ component we immediately see an increase in amplitude to a maximum which coincides with the maximum in the $b_{e}$ component. Our interpretation of this is that the increase seen in the $E_{r}$ component is the build-up of the toroidal mode. If we interpret the $6.8 \mathrm{mHz}$ wave as the fundamental mode oscillation along the geomagnetic field lines, we should have a node in the azimuthal magnetic field component at the equator and since SC 4 passes the magnetic equator at approximately 14:07 UT this would explain the very small amplitude observed in the $b_{e}$ component the first ten minutes after the onset. The increase in amplitude of this component would then be a combination of the satellite moving along the geomagnetic field lines, thus moving away from the node and also the buildup of the toroidal mode. However since this is at the onset of the event the question naturally arises if there has been enough time for a standing wave and thus a node to develop. Thus we need to examine the phase difference between the two components. We will address this question further down.

Figure 6 shows the fields measured by SC 3. Here we have not used the $\mathbf{E} \cdot \mathbf{B}=0$ assumption since the orientation of the spacecraft is such that the spin-plane of the satellite makes a very small angle with the background magnetic field just at the time of the onset of the event. The oscillations at the onset are dominantly in the radial direction of the magnetic field. Looking at the $b_{e}$ component we see that there is a minimum in amplitude coinciding with the spacecraft crossing the magnetic equator. This again is consistent with a node in the magnetic field at the equator, indicating an odd harmonic. The eastward electric field component shows persistent oscillations for a large portion of the time interval. During the time interval the oscillations are observed in the $E_{e}$ component SC 3 covers a range of $L$ values from approximately 5.5 at the onset of the event to a minimum of 4.3. Thus this poloidal mode oscillation has a relatively large radial extent. The dominant oscillation at the onset of the event is observed in the field-aligned component of the wave magnetic field for SC 1, 2 and 4. For SC 3 however the dominant oscillation is in the radial component.

For determining the azimuthal wave number for waves with small azimuthal wave number the technique used by Eriksson et al. (2005a) is not fully satisfactory. Instead we adopt a different approach in this paper. We take the duskward electric field measured by two satellites and calculate the cross spectral density (CSD). This is computed using Welch's (Welch, 1967) averaged, modified periodogram method, where we divide the data vector into eight segments with $50 \%$ overlap. From the CSD we can extract the phase difference as a function of frequency between the two signals. This approach was used by Takahashi et al. (1985). The azimuthal wave number is then calculated by dividing the phase difference with the azimuthal separation between the satellites. We compute the CSD from the time period 14:09 UT to 15:00 UT and use the phase difference at $6.8 \mathrm{mHz}$. Here we get high coherency $(>0.9)$ between the three satellites 1, 2 and 4 . For pairing with SC 3 we get a coherency in the range $0.6-0.7$. From the calculations of the CSD we obtain six different estimates of the wave number from the six different satellite pairs and obtain $m=10 \pm 3$. Since we observe two discrete frequencies, we can compute also the phase difference at $27 \mathrm{mHz}$. However since the $27 \mathrm{mHz}$ wave is present for a shorter time interval in the data we need to adjust the time period for which the CSD is computed. We use the time interval 14:09 UT to 14:25 UT. Again we get high coherency $(0.85-0.95)$ for the triplet 1,2 and 4 whereas for pairing with SC 3 it is quite poor $(<0.4)$. Despite the large coherency for the pairs $1-2,1-4$ and $2-4$ there is a large spread in the estimates of $m$ and we are unable to get 


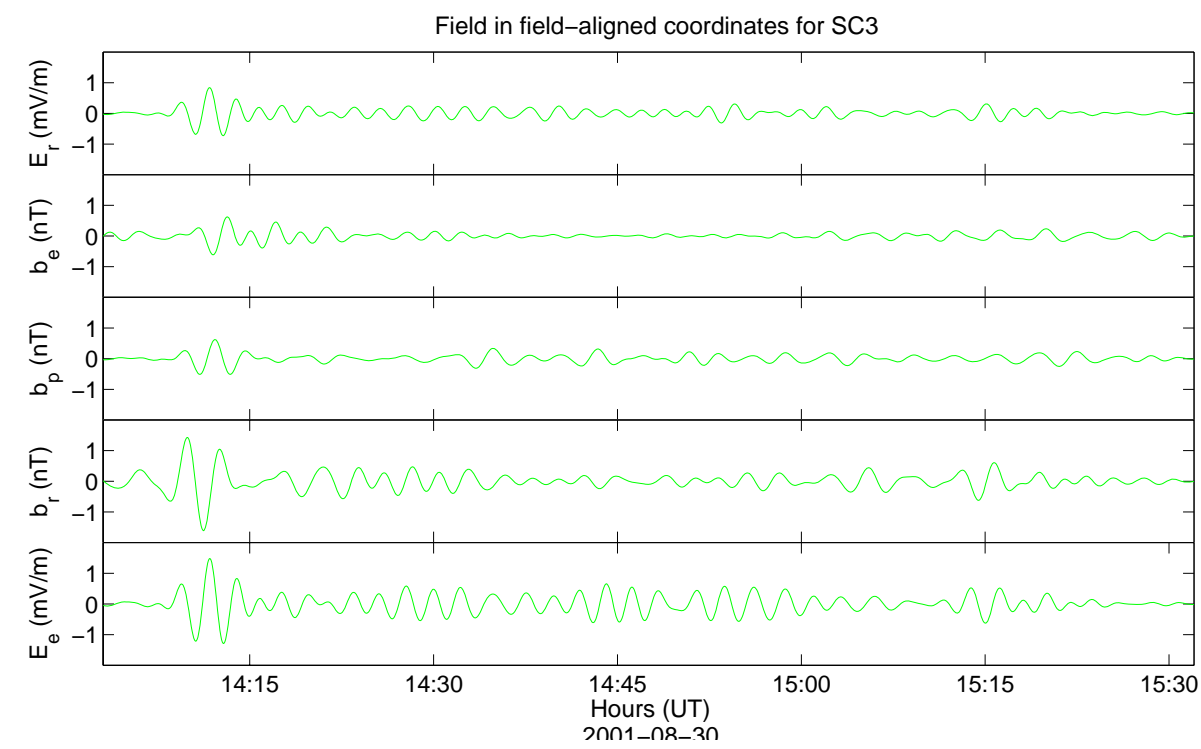

Fig. 6. Field in field-aligned coordinates measured by SC 3 . The data have been bandpass filtered in the range $4-9 \mathrm{mHz}$. Here we have not used the $\mathbf{E} \cdot \mathbf{B}=0$ assumption.

a consistent result. The estimates are notably larger than for the $6.8 \mathrm{mHz}$ wave though. We will discuss this later in the paper.

By looking at the phase difference between the electric and magnetic field components in the field-aligned coordinate system we can tell if the observed waves are propagating or standing waves (in phase or $90^{\circ}$ out of phase) along or across geomagnetic field lines. Figure 7 shows the band passed filtered (passband 4-9 $\mathrm{mHz}$ ) magnetic and electric field components measured by SC 4 . The three magnetic field components are plotted with their corresponding electric field components respectively and with the instantaneous phase difference (calculated from the analytic signal, e.g., Glassmeier, 1980) between the two components plotted below. In Fig. 7a we have plotted the parallel component of the magnetic field and the eastward component of the electric field. Figure $7 b$ shows the eastward component of the magnetic field and the radial component of the electric field. These two components correspond to the toroidal mode and although the amplitude is very small in the beginning for the $b_{e}$ component it can be seen that it is in phase with the $E_{r}$ component for the first two periods and then they rapidly become $90^{\circ}$ out of phase. In addition to looking at the phase difference between the two components, the question concerning the correlation of the amplitude is of interest. As the toroidal mode builds up the electric field amplitude increases more rapidly than the magnetic field amplitude. However, when the phase difference between the two signals have reached approximately $90^{\circ}$ the amplitudes remain closely correlated.

Figure $7 \mathrm{c}$ shows the phase difference between the radial component of the magnetic field and the eastward component of the electric field. These field components correspond to a poloidal mode type oscillation with Poynting flux along the magnetic field lines. For the first two wave periods the two components are in phase, indicating that this is a propagating wave. Then the phase difference rapidly changes as seen in the bottom panel of Fig. 7 and the components become approximately $90^{\circ}$ out of phase, which corresponds to a standing wave. Looking again at Fig. $7 \mathrm{~b}$ it is worth noting that the amplitudes of the two oscillations seem to be correlated after 14:30 UT. On the other hand, looking at Fig. 7a the amplitude of $E_{e}$ seems to correlate with the amplitude of $b_{p}$ at the beginning of the event. Theoretical studies (Chen and Hasegawa, 1974b; Allan et al., 1986; Lee and Lysak, 1989, 1991; Southwood and Kivelson, 1990) of ULF waves often interpret each of the three magnetic field components as representing a different mode. So the field-aligned component is interpreted as the global (waveguide) mode, the radial component as the poloidal mode and the eastward component as the toroidal mode. In reality this may be an oversimplification considering that the two electric field components are coupled with the three magnetic field components when looking at the MHD equations. Thus it is not totaly straightforward to compare $E_{e}$ with $b_{r}$ or $b_{p}$. However in this case we can speculate that the oscillations seen in the $E_{e}$ component at the beginning of the event correspond to oscillations in $b_{p}$ (compressional mode) and that the oscillations in $E_{e}$ after 14:30 UT are associated with $b_{r}$ (poloidal mode). The phase relationship between the $b_{p}$ and the $E_{e}$ component is complicated, which may be related to the close proximity of the plasmapause. In Fig. 7d we have plotted the parallel magnetic field component and the radial electric field component. Their amplitude and phase relationships are complicated throughout the time interval. 

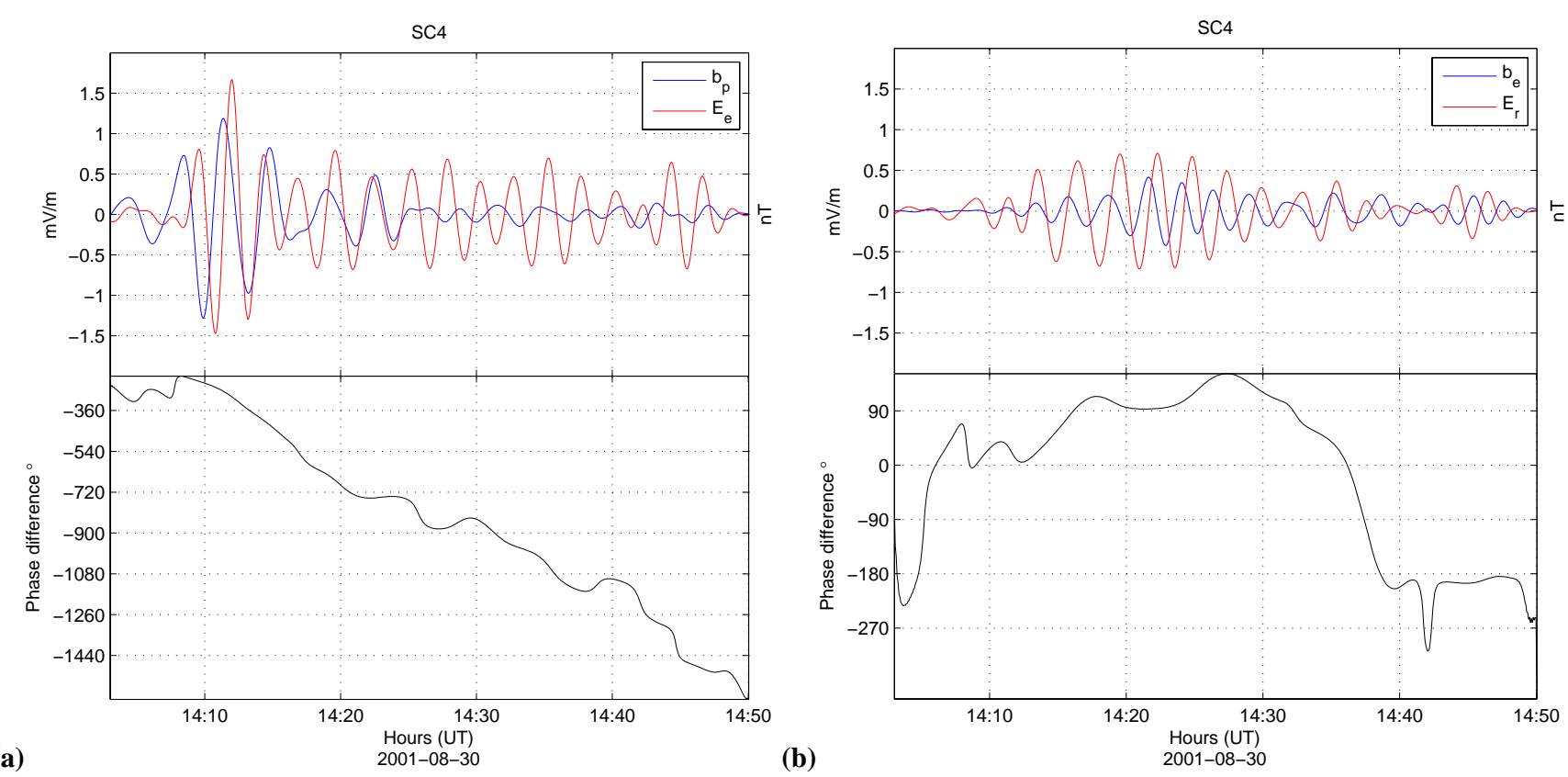

(a)

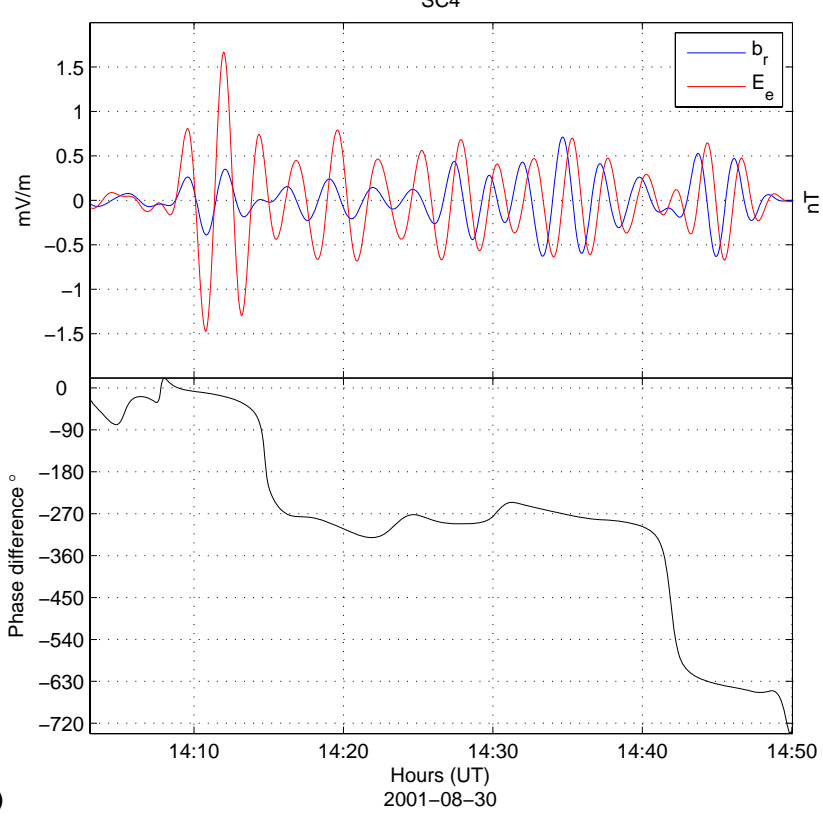

(b)

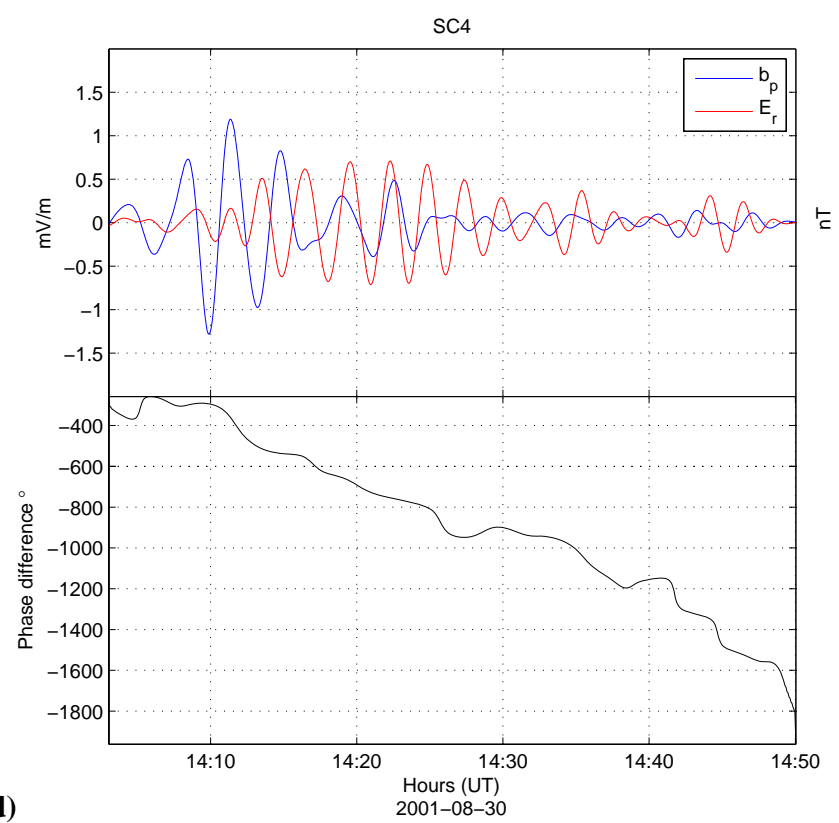

Fig. 7. The top panel in each figure shows one bandpass filtered (passband 4-9 mHz) component of the electric field (red) and one component of the magnetic field (blue) measured by SC 4. The bottom panel shows the instantaneous phase difference between these two components. (a) Shows the eastward electric field component and the parallel magnetic field component; (b) the eastward magnetic and radial electric field components; (c) eastward electric and radial magnetic field components; (d) parallel magnetic and radial electric components.

We now turn our attention to the $27 \mathrm{mHz}$ oscillation. In Fig. 8 we have band pass filtered the data with passband 22$32 \mathrm{mHz}$ to isolate the $27 \mathrm{mHz}$ wave. Figure 8 a shows the parallel magnetic field component and the eastward electric field component, corresponding to the compressional wave. The two components are approximately $180^{\circ}$ out of phase, which indicates a wave propagating anti-sunward. There also seems to be two wave packets involved here. The first one starting at the onset of the event and ending just after 14:15 UT followed by another one with significantly smaller amplitude but still with approximately $180^{\circ}$ phase shift. Figure $8 \mathrm{~b}$ shows the toroidal mode field components. Here we have the same behavior as for the $6.8 \mathrm{mHz}$ wave. Looking at the electric field component, we see an increase in amplitude 

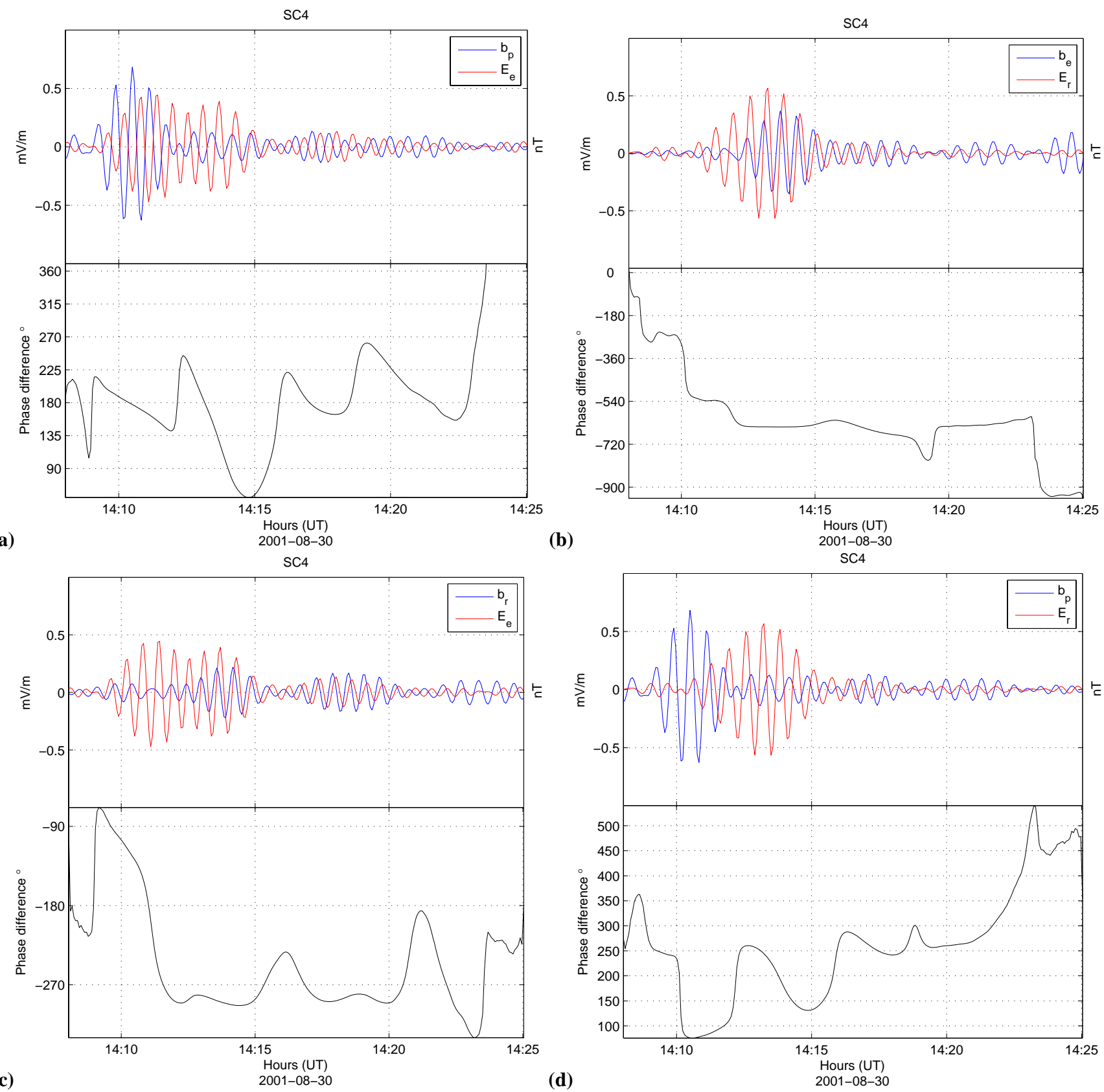

Fig. 8. Same as Fig. 7 but bandpass filtered with passband $22-32 \mathrm{mHz}$.

for the first five or six wave periods and then the amplitude starts to decrease. The magnetic field component does not show the same rate of increase as the electric field component. The phase difference between the two components is approximately $180^{\circ}$ for the first two or three wave periods and then it rapidly changes so that the two components are $90^{\circ}$ out of phase. Interestingly, as soon as the two components become $90^{\circ}$ out of phase the amplitude of the $b_{e}$ component increases rapidly and is then correlated with the amplitude of the radial electric field. For the $b_{r}$ component
(Fig. 8c) we see only small oscillations in the beginning of the event, again we might speculate that these are associated with the compressional mode. Like the $6.8 \mathrm{mHz}$ wave the $27 \mathrm{mHz}$ pulsation also has a complicated relationship between the parallel magnetic component and the radial electric component (Fig. 8d). The $b_{p}$ component oscillates for approximately two periods before the $E_{r}$ component starts to increase in amplitude. This increase continues even after the $b_{p}$ component starts to decrease. 

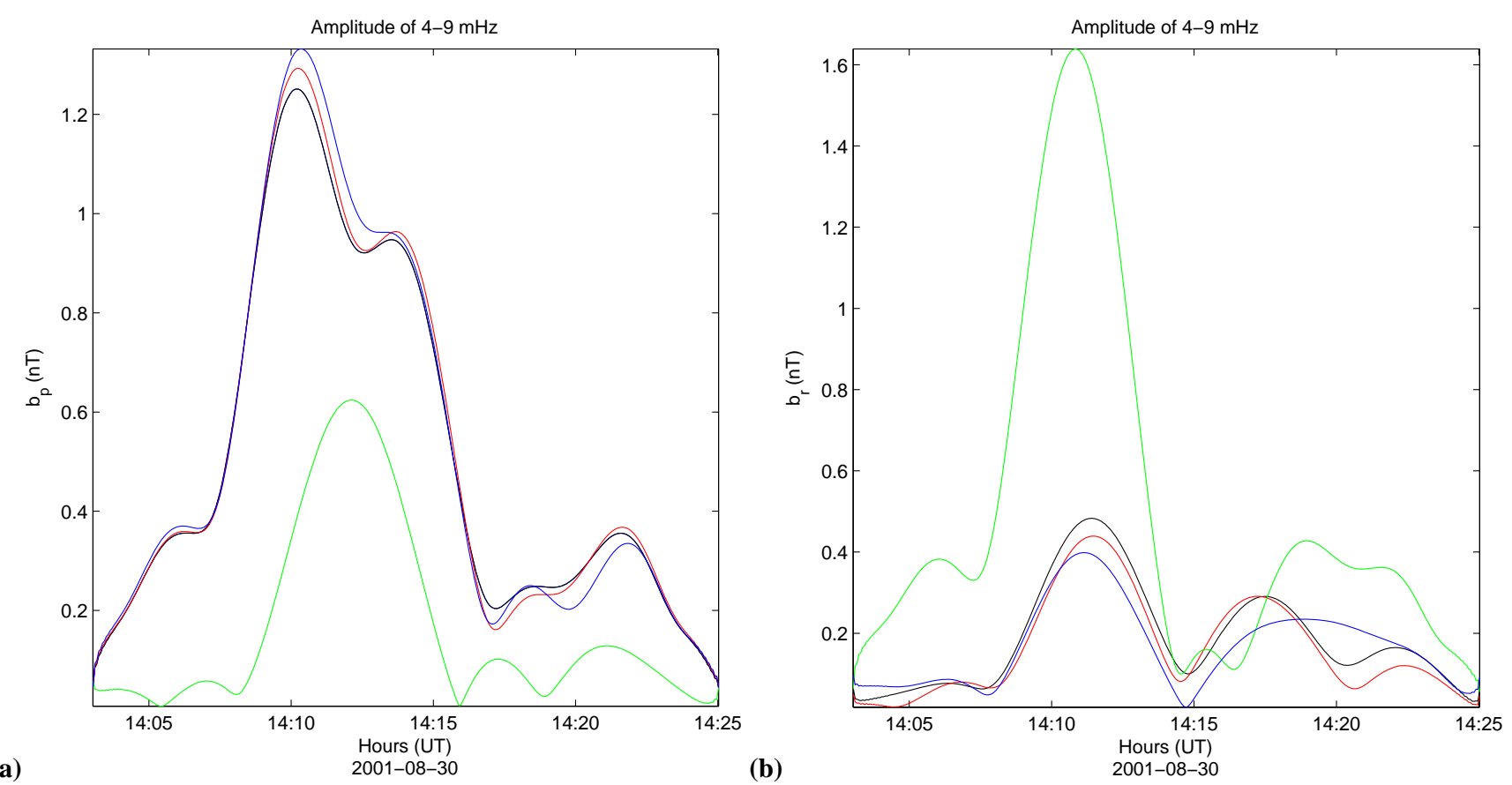

Fig. 9. Amplitude of the $b_{p}$ component (a) and the $b_{r}$ component (b) for the $6.8 \mathrm{mHz}$ wave.
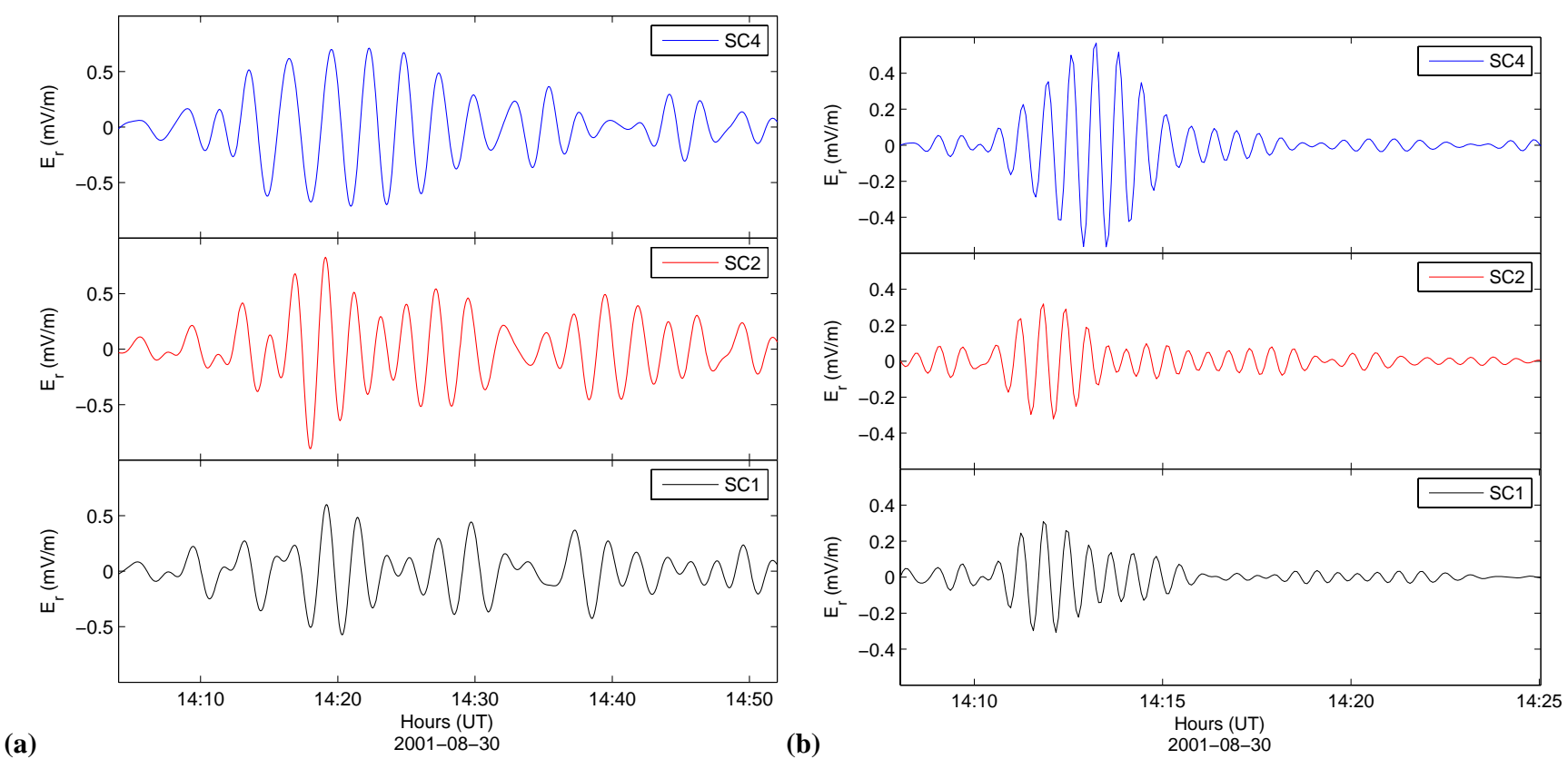

Fig. 10. The radial component of the electric field for SC 1 (black), SC 2 (red) and SC 4 (blue) for the (a) $6.8 \mathrm{mHz}$ wave (b) $27 \mathrm{mHz}$ wave.

In Fig. 9 we have plotted the instantaneous amplitude of the $b_{p}$ and $b_{r}$ components of the $6.8 \mathrm{mHz}$ wave for the time interval 14:02 UT to 14:25 UT. Here we have obtained the instantaneous amplitude by computing the analytic signal for the bandpass filtered data. Thus, these plots illustrate the wave packet structure of the impinging global mode wave. Here we clearly see again the difference in polarisation between SC 3 and the other spacecraft. Furthermore there is a small difference between the triplet 1, 2 and 4. SC 4, which is closest to the magnetic equator at the time of onset has 
the largest maximum amplitude in the $b_{p}$ component and the smallest maximum amplitude in the $b_{r}$ component. For SC 1 , which is furthest away from the equator at the time of onset, we have the smallest maximum amplitude in the $b_{p}$ component of the three satellites, but the largest maximum in the $b_{r}$ component. Now the separation in $L$ between the spacecraft for the time interval plotted is approximately $0.15 R_{E}$ for SC 1 and 2 and the same for SC 2 and 4 . If the compressional wave is propagating Earthward we should expect the wave packets observed to be separated in time by $0.15 R_{E} / v_{g}$, where $v_{g}$ is the group velocity of the wave. Unfortunately, even for moderate group velocities (Mann et al., 1998, estimated $v_{g} \sim 220 \mathrm{~km} / \mathrm{s}$ for an observed waveguide mode) the separation in time is much smaller than the wave period and thus it would be hard to get an accurate estimate.

Figure 10 shows the behavior of the toroidal pulsations measured by SC 1,2 and 4 , both for the $6.8 \mathrm{mHz}$ and the $27 \mathrm{mHz}$ wave. Since SC 3 is approximately $30 \mathrm{~min}$ "after" the other satellites it is hard to compare the amplitude observed by SC 3 with the other since the waves have had time to dampen significantly. Here we see that SC 4 observes the largest amplitude for both of the frequencies and that the amplitude decreases with increasing $L$-value.

\section{Discussion}

Here we present observation of the simultaneous excitation of both a toroidal and a poloidal mode by a magnetospheric waveguide mode, which in turn has been generated by a sudden enhancement in the solar wind dynamic pressure. Both the toroidal and poloidal mode begin as travelling waves and transform into standing waves along the geomagnetic field lines. We estimated the azimuthal wave number to be 10 for the $6.8 \mathrm{mHz}$ and significantly larger for the $27 \mathrm{mHz}$ wave. With these relatively large values of $m$ we have to consider that the toroidal and poloidal oscillations are coupled. Also as seen from the difference in polarisation between the different satellites, it is not totally straightforward to interpret the oscillations in the $b_{r}$ component as being either part of a global waveguide mode or a poloidal mode. Unfortunately, this is something that has received little attention in the literature. There are some papers that discuss a poloidal mode FLR, e.g. Ding et al. (1995), Fenrich et al. (1995), Cramm (2000), but their simultaneous occurrence in conjunction with a cavity or waveguide mode have to the authors' knowledge never received much attention. Usually, oscillations in the $b_{r}$ component (or $b_{x}$ in the box-model often used, e.g. Walker, 2002) are associated with the global cavity or waveguide mode.

Several analytical and numerical studies have shown that energy from the solar wind most efficiently drives field line resonances for $m=3$ (Allan et al., 1986, in the case of the magnetospheric cavity model; Walker, 2000, for the waveguide model). A single value for the azimuthal wave number implies that the source occurs coherently around the entire magnetosphere. In reality the source may be localised to a part of the magnetosphere or at least in the case of impulse excitation we could expect $m$ to be larger than 3. Indeed, an enhancement in the solar wind pressure would create a ripple on the magnetopause propagating anti-sunward as demonstrated by Elphic (1989). The dimension (or wavelength) of this ripple is determined by the time scale of the enhancement in pressure, the magnitude of the enhancement and of the radius of the magnetosphere. From this a coarse estimate of $m$ can be made (e.g. Mann et al., 1998) by relating the scale size of the ripple to the azimuthal wavelength and thus also to the azimuthal wave number. Since the magnetopause standoff distance $R_{m p}$ scales as $R_{m p} \propto p_{\text {dyn }}^{-1 / 6}$, a threefold increase in the dynamic pressure only gives a $20 \%$ decrease in the standoff distance. Assuming that the initial standoff distance was $10 R_{E}$ we should expect a scale size of the disturbance in the anti-sunward direction of approximately $2 R_{E}$. The second scale size to consider is determined by the duration of the event and the propagation speed of the disturbance at the magnetopause. This propagation speed is the sum of the fluid flow speed and the characteristic information speed in the magnetosheath. Interestingly, Kaymaz et al. (1995) noted that if the magnetic pressure is neglected, the sum of the sound speed and the fluid speed in the magnetosheath is generally very close to the solar wind speed. Thus we assume for simplicity that the propagation speed of the ripple along the magnetopause is the same as the solar wind speed. We can write $\lambda=v_{\text {prop }} \tau$ for the wavelength of the disturbance at the magnetopause, where $v_{\text {prop }}=500 \mathrm{~km} / \mathrm{s}$ and $\tau=120 \mathrm{~s}$ (from the rise-time of the solar wind pressure) for this event. As a first approximation we can assume that the scale size of the ripple is largely determined by the time scale of the pressure enhancement. For the azimuthal wave number we can write $m=k_{y} R_{m p}$ with $k_{y}=2 \pi / \lambda$ and we get $m \approx 7$ which is in relatively good agreement with the estimate from Cluster for the $6.8 \mathrm{mHz}$ wave. So although $m=3$ theoretically gives the best coupling of energy, there is reason to allow other values of $m$ in future studies of the magnetosphere. Lee and Lysak (1991) took this into account that $m=3$ might not always accurately describe realistic conditions and studied impulse excitation of a three dimensional model of the magnetosphere where they varied the azimuthal wave number. They concluded that as the longitudinal width of the impulse becomes larger at the magnetopause the field line resonances generated are more likely to have smaller $m$ numbers. For a more narrow impulse the waves generated in general have larger values of $m$. This treatment of the magnetospheric response to an impulse by Lee and Lysak (1991) is in the spirit of the cavity mode theory. In the case of our observations here we have a disturbance that is propagating along the magnetopause. It thus seems more appropriate to compare the observations with the waveguide mode theory. We again turn the attention to our observations of 
the azimuthal wave number. From the CSD we observed that the phase difference between the satellite pairs were significantly larger at $27 \mathrm{mHz}$ than at $6.8 \mathrm{mHz}$. This might indicate that the phase difference at this frequency has contributions from other factors than the azimuthal separation, such as the radial separation. However, since the phase difference is consistently several times larger we might speculate on a different underlying effect. In a box-model simulation of the magnetospheric waveguide by Wright and Rickard (1995), two types of excitation mechanisms were investigated. The first case, called a standing pulse, corresponded to a displacement of the magnetopause over a fixed azimuthal range. In this case the resulting pulsations were shown to have phase speeds and azimuthal wave numbers determined by the equilibrium structure of the magnetosphere. The second case, called a running pulse, constituted a ripple travelling antisunward along the magnetopause. The pulsations driven by this mechanism were shown to have a phase velocity strictly equal to that of the ripple. These theoretical predictions were elaborated into a diagnostics scheme by Mann and Wright (1999) (their Fig. 2), that was applied ground-based magnetometer data by Mathie and Mann (2000). If we write the azimuthal phase speed of the wave as $V_{p}=\left|2 \pi L R_{E} f / m\right|$, where $R_{E}$ is the radius of the Earth and $f$ is the wave frequency, we should expect the ratio $f / m$ to be constant for FLRs observed on the same $L$-value that are being driven by a running pulse. If the two different toroidal pulsations observed have the same phase velocity, we should expect the $27 \mathrm{mHz}$ wave to have an azimuthal wave number and thus also a phase difference four times larger than the $6.8 \mathrm{mHz}$ wave. The poor coherency obtained when calculating the CSD from SC 3 paired with any of the other for the $27 \mathrm{mHz}$ wave is in contrast to the coherency for the $6.8 \mathrm{mHz}$ wave which is in the range 0.6-0.7. This might be explained by the higher frequency wave having a smaller scale size. This smaller scale size might also explain why we fail to determine $m$ for the $27 \mathrm{mHz}$ wave but get a good estimate for the $6.8 \mathrm{mHz}$ wave.

The difference in polarisation in the magnetic field observed by SC 1, 2, 4 and SC 3 can be understood in terms of the field line displacement. With Faraday's law and the frozen-in condition, we get an equation for the magnetic field and the plasma motion as

$$
\frac{d \mathbf{B}}{d t}=(\mathbf{B} \cdot \nabla) \mathbf{v}-\mathbf{B} \nabla \cdot \mathbf{v} .
$$

As SC 1, 2 and 4 are close to the magnetic equator at the time of onset the plasma displacement at their locations can be assumed to be perpendicular to the magnetic field lines; at the location of SC 3 on the other hand, the field lines are to a first approximation parallel to the motion of the plasma. We assume a perturbation $\mathbf{b}_{1}$ to the magnetic field as $\mathbf{B}=\mathbf{B}_{0}+\mathbf{b}_{1}$, where $\mathbf{B}_{0}$ is the background magnetic field. The first term in the right hand side of Eq. (1) is the gradient of $\mathbf{v}$ along the field lines. Since we assumed that the field line displace- ment is perpendicular to the background magnetic field at the equator this term vanishes and we get

$$
\frac{d \mathbf{b}_{1}}{d t}=-\mathbf{B}_{0} \nabla \cdot \dot{\xi}
$$

where $\dot{\xi}$ is the time derivative of the plasma displacement. Thus at the location of SC 1, 2 and 4 the perturbation of the magnetic field is mainly in the field-aligned direction. SC 3 is located approximately $20^{\circ}$ south of the magnetic equator and as apparent from Fig. 1 we cannot assume that the plasma displacement is perpendicular to the background magnetic field at this position. Instead we have for the term $(\mathbf{B} \cdot \nabla) \mathbf{v}=\left(\left[\mathbf{B}_{0}+\mathbf{b}_{1}\right] \cdot \nabla\right) \dot{\xi} \approx\left(\mathbf{B}_{0} \cdot \nabla\right) \dot{\xi}$ to lowest order. Depending on the direction of $\dot{\boldsymbol{\xi}}$ we can have oscillation in the perpendicular directions as well and the polarisation can have a more complicated form, even to lowest order.

Interestingly there are not any transient waves at several different frequencies observed, rather the $6.8 \mathrm{mHz}$ and the $27 \mathrm{mHz}$ pulsations are present from the beginning. Thus it seems unlikely that the waves observed by Cluster are directly caused by the pressure enhancement; that is broadband to its nature; but rather the geomagnetic waveguide acts as a frequency filter (or optical filter as suggested by Walker, 2002). Of the frequencies "selected" by the magnetosphere (the waveguide eigenfrequencies) Cluster detects two, one at $6.8 \mathrm{mHz}$ and another at $27 \mathrm{mHz}$. Of course, lower frequency waves might also have been generated, but these would most likely drive field line resonances at larger $L$-values.

The model by Carpenter and Anderson (1992) suggests that the observed toroidal mode pulsation is located within the plasmasphere. Unfortunately, we do not have any precise measurement of the density for this time interval and thus cannot verify this assumption. The poloidal mode pulsations however are observed beyond the model plasmapause. On the other hand, the plasmapause would be a natural place for a FLR to develop due to higher phase-mixing rate (e.g. Wright and Allan, 1996, 1999).

Since the toroidal mode for both the $6.8 \mathrm{mHz}$ and the $27 \mathrm{mHz}$ wave is observed to have the largest amplitude at SC 4 and that the amplitude decreases for SC 2 and 1 that are located on larger $L$-shells, we interpret these waves as field line resonances Earthward of the satellites. Simultaneously we have a poloidal mode that is present over a much larger range of $L$-values. These two modes are excited by the cavity/waveguide mode that is present only for a couple of wave periods. This could be interpreted as the waveguide mode propagating tailward in the same manner as observed by Mann et al. (1998).

Energy in the $b_{p}$ component decreases before the increase in the $b_{e}$ component has stopped. Rickard and Wright (1995) showed that the absence of coherent fast mode oscillations does not contradict the waveguide model. The time where the amplitude of $b_{r}$ and $E_{e}$ starts to correlate agrees relatively well with the time when the toroidal mode starts to decay. 


\section{Summary and conclusions}

We have studied the excitation of geomagnetic ULF waves by an enhancement in the solar wind dynamic pressure. Cluster observes two discrete frequencies, $6.8 \mathrm{mHz}$ and $27 \mathrm{mHz}$, which we interpret as two harmonics of the geomagnetic waveguide. For both frequencies the initial oscillations are mainly seen in the compressional magnetic field component by SC 1, 2 and 4 and in the radial magnetic field component by SC 3 . This is followed by oscillations in the toroidal and poloidal magnetic field components, both of which are travelling waves in the beginning but later develop into standing waves. For the toroidal mode this transformation occurs within approximately two wave periods for both the $6.8 \mathrm{mHz}$ and the $27 \mathrm{mHz}$ oscillation. For the poloidal mode the behavior is more complex and we argue that the oscillations in the radial component of the magnetic field and eastward component of the electric field are related to both the global magnetospheric waveguide mode and to a poloidal mode standing along the geomagnetic field lines. The toroidal mode for both frequencies have the largest coherency observed by SC 4 and then this coherency decreases with satellites that are located on higher $L$-values, indicating that this mode is localised in the radial direction as is expected for a field line resonance. The poloidal mode standing wave is observed over a much larger radial distance. We estimate the azimuthal wave number to be $10 \pm 3$ for the $6.8 \mathrm{mHz}$ wave. We are unable to determine an accurate estimate for the $27 \mathrm{mHz}$ wave but it is likely to be several times larger than the wave number for the $6.8 \mathrm{mHz}$ wave. This discrepancy can be explained by the two waves having the same phase velocity which results from the geomagnetic waveguide being excited by an impulse as shown by Wright and Rickard (1995). This paper also illustrated the need to further develop the magnetospheric waveguide model using more realistic geometries.

Acknowledgements. Work at the Royal Institute of Technology was partially supported by the Swedish National Space Board and the Alfvén Laboratory Centre for Space and Fusion Plasma Physics. Work at TU Braunschweig was financially supported by the German Bundesministerium für Wirtschaft und Technologie and the Deutsches Zentrum für Luft- und Raumfahrt under contract 50OC 0104.

Topical Editor I. A. Daglis thanks P. Francia and another referee for their help in evaluating this paper.

\section{References}

Allan, W., White, S. P., and Poulter, E. M.: Impulse-excited hydromagnetic cavity and field-line resonances in the magneosphere, Planet. Space Sci., 34, 371-385, 1986.

Baumjohann, W., Junginger, H., Haerendel, G., and Bauer, O. H.: Resonant Alfvén waves excited by a sudden impulse, J. Geophys. Res., 89, 2765-2769, 1984.

Cahill, L. J., Lin, N. G., Waite, J. H., Engebretson, M. J., and Sugiura, M.: Toroidal standing waves excited by a storm sud- den commencement: DE 1 observations, J. Geophys. Res., 95, 7857-7867, 1990 .

Carpenter, D. L. and Anderson, R. R.: An ISEE/Whistler model of equatorial electron density in the magnetosphere, J. Geophys Res., 97, 1097-1108, 1992.

Chen, L. and Hasegawa, A.: A theory of long-period magnetic pulsations, 1, Steady state excitation of field line resonance, J. Geophys. Res., 79, 1024-1032, 1974a.

Chen, L. and Hasegawa, A.: A theory of long-period magnetic pulsations. II. Impulse excitation of surface eigenmode, J. Geophys Res., 79, 1033-1037, 1974b.

Chi, P. J., Russell, C. T., Raeder, J., et al.: Propagation of the preliminary reverse impulse of sudden commencements to low latitudes, J. Geophys. Res., 106, 18 857-18 864, 2001.

Cramm, R., Glassmeier, K. H., Othmer, C., Fornacon, K. H., Auster, H. U., Baumjohann, W., and Georgescu, E.: A case study of a radially polarized Pc4 event observed by the Equator-S satellite, Ann. Geophys., 18, 411-415, 2000, http://www.ann-geophys.net/18/411/2000/.

Ding, D. Q., Denton, R. E., Hudson, M. K., and Lysak, R. L.: An MHD simulation study of the poloidal mode field line resonance in the Earth's magnetosphere, J. Geophys. Res., 100, 63 $78,1995$.

Elphic, R. C.: Multipoint observations of the magnetopause: Results from ISEE and AMPTE, Adv. Space Res., 8, 223-238, 1988.

Eriksson, P. T. I., Blomberg, L. G., and Glassmeier, K.-H.: Cluster satellite observations of $\mathrm{mHz}$ pulsations in the dayside magnetosphere, Adv. Space Res., doi:10.1016/j.asr.2005.04.103, in press, 2005a.

Eriksson, P. T. I., Blomberg, L. G., Walker, A. D. M., and Glassmeier, K.-H.: Poloidal ULF oscillations in the dayside magnetosphere: a Cluster study, Ann. Geophys., 23, 2679-2686, 2005 b.

Fenrich, F. R., Samson, J. C., Sofko, G., and Greenwald, R. A.: ULF high- and low-m field line resonances observed with the Super Dual Auroral Radar Network, J. Geophys. Res., 100, 21535 $21548,1995$.

Glassmeier, K.-H.: Magnetometer array observations of a giant pulsation event, J. Geophys, 48, 127-138, 1980.

Goldstein, J., Hudson, M. K., and Lotko, W.: Possible evidence of damped cavity mode oscillations stimulated by the January, 1997 magnetic cloud event, Geophys. Res. Lett., 26, 3589-3592, 1999.

Kaymaz, Z., Petschek, H. E., Siscoe, G. L., Frank, L. A., Ackerson, K. L., and Paterson, W. R.: Disturbance propagation times to the far tail, J. Geophys. Res., 100, 23 743-23 748, 1995.

Kivelson, M. G, Etcheto, J., and Trotignon, J. G.: Global compressional oscillations of the terrestrial magnetosphere: the evidence and a model, J. Geophys. Res., 89, 9851-9856, 1984.

Kivelson, M. G. and Southwood, D. J.: Resonant ULF waves: A new interpretation, Geophys. Res. Lett., 12, 49-52, 1985.

Kivelson, M. G. and Southwood, D. J.: Coupling of global magnetospheric eigenmodes to field line resonances, J. Geophys. Res., 91, 4345-4351, 1986.

Lee, D.-H. and Lysak, R. L.: Magnetospheric ULF wave coupling in the dipole model: the impulsive excitation, J. Geophys. Res., 94, 17 097-17 103, 1989.

Lee, D.-H. and Lysak, R. L.: Impulsive excitation of ULF waves in the three-dimensional dipole model: the initial results, J. Geo- 
phys. Res., 96, 3479-3486, 1991.

Mann, I. R., Chisham, G., and Bale, S. D.: Multisatellite and ground-based observations of a tailward propagating Pc5 magnetospheric waveguide mode, J. Geophys. Res., 103, 4657-4669, 1998.

Mann, I. R. and Wright, A. N.: Diagnosing the excitation mechanisms of Pc5 magnetospheric flank waveguide modes and FLRs, Geophys. Res. Lett., 26, 2609-2612, 1999.

Mann, I. R., Voronkov, I., Dunlop, M., Donovan, E., Yeoman, T. K., Milling, D. K., Wild, J., Kauristie, K., Amm, O., Bale, S. D., Balogh A., Viljanen A., and Opgenoorth, H. J.: Coordinated ground-based and Cluster observations of large amplitude global magnetospheric oscillations during a fast solar wind speed interval, Ann. Geophys., 20, 405-426, 2002,

http://www.ann-geophys.net/20/405/2002/.

Mathie, R. A. and Mann, I. R.: Observations of Pc5 field line resonance azimuthal phase speeds: A diagnostic of their excitation mechanism, J. Geophys. Res., 105, 10 713-10 728, 2000.

Nopper, R. W., Hughes, W. J., McLennan, C. G., and McPherron, R. L.: Impulse-excited pulsations during the July 29, 1977, event, J. Geophys. Res., 87, 5911-5916, 1982.

Radoski, H. R.: A theory of latitude dependent geomagnetic micropulsations: the asymptotic fields, J. Geophys. Res., 79, 595603, 1974.

Rae, I. J., Donovan, E. F., Mann, I. R., et al.: Evolution and characteristics of global Pc5 ULF waves during a high solar wind speed interval, J. Geophys. Res., 110, doi:10.1029/2005JA011007, 2005.

Rickard, G. J. and Wright, A. N.: ULF pulsations in a magnetospheric waveguide: Comparison of real and simulated satellite data, J. Geophys. Res., 100, 3531-3537, 1995.

Shimazu, H., Araki, T., Kamei, T., and Hanado, H.: A Symmetric Appearance of Pc5 on Dawn and Dusk Sides Associated with Solar Wind Pressure Enhancement, J. Geomag. Geoelectr., 47, 177-189, 1995.

Southwood, D. J.: Some features of field line resonances in the magnetosphere, Planet. Space Sci., 22, 483-491, 1974.

Southwood, D. J. and Kivelson, M. G.: The magnetohydrodynamic response of the magnetospheric cavity to changes in the solar wind pressure, J. Geophys. Res., 95, 2301-2309, 1990.

Takahashi, K., Higbie, P. R., and Baker, D. N.: Azimuthal propagation and frequency characteristic of compressional Pc 5 waves observed at geostationary orbit, J. Geophys. Res., 90, 1473$1485,1985$.
Tamao, T.: Transmission and coupling resonance of hydromagnetic disturbaces in the non-uniform Earth's magnetosphere, Science Reports of Tohoku University, Series 5, Geophysics, 43, 43-72, 1965.

Tsyganenko, N. A.: A magnetospheric magnetic field model with a warped tail current sheet, Planet. Space Sci, 37, 5-20, 1989.

Walker, A. D. M., Ruohoniemi, J. M., Baker, K. B., Greenwald, R. A., and Samson, J. C.: Spatial and temporal behavior of ULF pulsations observed by the Goose Bay HF radar, J. Geophys. Res., 97, 12 187-12 202, 1992.

Walker, A. D. M.: Excitation of magnetohydrodynamic cavities in the magnetosphere, J. Atmos. Solar Terrest. Phys., 60, 12791293, 1998.

Walker, A. D. M.: Coupling between waveguide modes and field line resonances, J. Atmos. Solar Terrest. Phys., 62, 799-813, 2000.

Walker, A. D. M.: Excitation of field line resonances by MHD waves originating in the solar wind, J. Geophys. Res., 107(A12), 1481, doi:10.1029/2001JA009188, 2002.

Walker, A. D. M.: Excitation of field line resonances by sources outside the magnetosphere, Ann Geophys., 23, 3375-3388, 2005.

Waters, C. L., Takahashi, K., Lee, D.-H., and Anderson, B. J.: Detection of ultralow-frequency cavity modes using spacecraft data, J. Geophys. Res., 107(A10), 1284, doi:10.1029/2001JA000224, 2002.

Welch, P. D.: The Use of Fast Fourier Transform for the Estimation of Power Spectra: A Method Based on Time Averaging Over Short, Modified Periodograms, IEEE Trans. Audio Electroacoust., vol. AU-15, 70-73, 1967.

Wright, A. N.: Dispersion and wave coupling in inhomogeneous MHD waveguides, J. Geophys. Res., 99, 159-167, 1994.

Wright, A. N. and Rickard, G. J.: ULF pulsations driven by magnetopause motions: azimuthal phase characteristics, J. Geophys. Res., 100, 23 703-23 710, 1995a.

Wright, A. N. and Rickard, G. J.: A numerical study of resonant absorption in a magnetohydrodynamic cavity driven by a broad band spectrum, Astrophys. J., 444, 458-470, 1995 b.

Wright, A. N. and Allan, W.: Structure, phase motion, and heating within Alfven resonances, J. Geophys. Res., 101, 17 399-17 408, 1996.

Wright, A. N., Allan, W., Elphinstone, R. D., and Cogger, L. L.: Phase mixing and phase motion of Alfven waves on tail like and dipole like magnetic field lines, J. Geophys. Res., 104, 10159 $10175,1999$. 